\title{
Best approximation of functions in generalized Hölder class
}

H.K. Nigam ${ }^{1 *}$ and Md. Hadish ${ }^{1 \dagger}$

${ }^{*}$ Correspondence:

hknigam@cusb.ac.in

${ }^{1}$ Department of Mathematics, Central University of South Bihar,

Gaya, India

†Equal contributors

\begin{abstract}
Here, for the first time, error estimation of the functions $g \in H_{z}^{(w)}$ and $\tilde{g} \in H_{z}^{(w)}$ classes using TC ${ }^{1}$ method of F. S. (Fourier Series) and C. F. S. (Conjugate Fourier Series), respectively, are determined. The results of (Dhakal in Int. Math. Forum 5(35):1729-1735, 2010; Dhakal in Int. J. Eng. Technol. 2(3):1-15, 2013; Kushwaha and Dhakal in Nepal J. Sci. Technol. 14(2):117-122, 2013) become the particular cases of our Theorem 2.1. Some important corollaries are also deduced from our main theorems.
\end{abstract}

MSC: 41A10; 41A25; 42B05; 42A50; 40G05; 40C05

Keywords: Best approximation; Generalized Hölder class; Matrix ( $T$ ) means; $C^{1}$ means; $T^{1}$ means; Fourier series; Conjugate Fourier series

\section{Introduction}

Several results on the error estimation of a function $g$ in Lipschitz and Hölder classes by a trigonometric polynomial using different single and product means have been obtained by the researchers like [1-11], and [12].

Our motivation for this work is to consider a more advanced class of functions that can provide best approximation by a trigonometric polynomial of degree not more than $r$. Therefore, in this work, we generalize the results of Kushwaha and Dhakal [3] and Dhakal $[1,2]$. In fact, we obtain the results on the error estimation for the function $f \in H_{z}^{(w)}(z \geq 1)$ by T.C $C^{1}$ method by F. S. Thus, the results of Kushwaha and Dhakal [3] and Dhakal [1,2] become the particulars cases of our Theorem 2.1.

We also obtain the results on the error estimation of the function $\tilde{g} \in H_{z}^{(w)}(z \geq 1)$ by T.C ${ }^{1}$ method of C. F. S.

Let " $T=\left(a_{r, m}\right)$ be an infinite triangular matrix satisfying the conditions of regularity [13], i.e.,

$$
\begin{aligned}
& \sum_{m=0}^{r} a_{r, m}=1 \quad \text { as } r \rightarrow \infty \\
& a_{r, m}=0 \quad \text { for } m>r \\
& \sum_{m=0}^{r}\left|a_{r, m}\right| \leq M, \quad \text { a finite constant. }
\end{aligned}
$$

(c) The Author(s) 2018. This article is distributed under the terms of the Creative Commons Attribution 4.0 International License (http://creativecommons.org/licenses/by/4.0/), which permits unrestricted use, distribution, and reproduction in any medium, provided you give appropriate credit to the original author(s) and the source, provide a link to the Creative Commons license, and indicate if changes were made. 
The sequence-to-sequence transformation

$$
t_{r}^{T}:=\sum_{m=0}^{r} a_{r, m} s_{m}=\sum_{m=0}^{r} a_{r, r-m} s_{r-m}
$$

defines the sequence $t_{r}^{T}$ of triangular matrix means of the sequence $\left\{s_{r}\right\}$ generated by the sequence of coefficients $\left(a_{r, m}\right)$.

If $t_{r}^{T} \rightarrow s$ as $r \rightarrow \infty$, then the infinite series $\sum_{r=0}^{\infty} h_{r}$ or the sequence $\left\{s_{r}\right\}$ is summable to $s$ by a triangular matrix ( $T$-method) [14]."

"Let

$$
\begin{aligned}
C_{r}^{1} & =\frac{s_{0}+s_{1}+\cdots+s_{r}}{r+1} \\
& =\frac{1}{r+1} \sum_{m=0}^{r} s_{m} \rightarrow s \quad \text { as } r \rightarrow \infty .
\end{aligned}
$$

If $C_{r}^{1} \rightarrow s$ as $r \rightarrow \infty$, then the infinite series $\sum_{r=0}^{\infty} h_{r}$ is summable to $s$ by $C^{1}$ means [14]." The $T C^{1}$ means (T-means of $C^{1}$ means) is given by

$$
\begin{aligned}
t_{r}^{T . C^{1}} & :=\sum_{m=0}^{r} a_{r, m} C_{m}^{1} \\
& =\sum_{m=0}^{r} a_{r, m} \frac{1}{m+1} \sum_{v=0}^{m} s_{m} .
\end{aligned}
$$

If $t_{r}^{T . C^{1}} \rightarrow s$ as $r \rightarrow \infty$, then the series $\sum_{r=0}^{\infty} h_{r}$ or the sequence $\left\{s_{r}\right\}$ is summable to $s$ by T. $C^{1}$ means.

The regularity of $T$ and $C^{1}$ methods implies the regularity of $T \cdot C^{1}$ method.

Remark 1 (Example) Consider an infinite series

$$
1+\sum_{n=1}^{\infty}(-1)^{n} \cdot 2 n .
$$

The $n$th partial sum of (5) is given by

$$
s_{n}= \begin{cases}n+1, & n \text { is even } \\ 0, & n \text { is odd }\end{cases}
$$

and so

$$
C_{n}^{1}= \begin{cases}1, & n \text { is even, } \\ 0, & n \text { is odd }\end{cases}
$$

Therefore, series $(5)$ is not summable by $(C, 1)$ means.

If we take $a_{n, k}=\frac{1}{n+1}$, then series (5) is also not summable by $T$ means. But series (5) is summable by $T . C^{1}$ means. So, the product means is more powerful than the individual means. 
Remark $2 T C^{1}$ means reduces to

(i) $\left(H, \frac{1}{r+1}\right) C^{1}$ or $H \cdot C^{1}$ means if $a_{r, m}=\frac{1}{(r-m+1) \log (r+1)}$;

(ii) $\left(N, p_{r}\right) C^{1}$ or $N_{p} C^{1}$ means if $a_{r, m}=\frac{p_{r-m}}{P_{r}}$, where $P_{r}=\sum_{m=0}^{r} p_{m} \neq 0$;

(iii) $(N, p, q)(C, 1)$ or $N_{p, q} C^{1}$ means if $a_{r, m}=\frac{p_{r-m} q_{m}}{R_{r}}$, where $R_{r}=\sum_{m=0}^{r} p_{m} q_{r-m}$;

(iv) $\left(\bar{N}, p_{r}\right)(C, 1)$ or $\bar{N}_{p} C^{1}$ means if $a_{r, m}=\frac{p_{m}}{P_{r}}$.

Let $L^{z}[0,2 \pi]=\left\{g:[0,2 \pi] \rightarrow \mathbb{R}: \int_{0}^{2 \pi}|g(x)|^{z} d x<\infty, z \geq 1\right\}$ be the space of functions $(2 \pi$ periodic and integrable). We define the norm $\|\cdot\|_{(z)}$ by

$$
\left\{\frac{1}{2 \pi} \int_{0}^{2 \pi}|g(x)|^{z} d x\right\}^{\frac{1}{z}}, \quad z \geq 1
$$

As defined in "[14], $w:[0,2 \pi] \rightarrow \mathbb{R}$ is an arbitrary function with $w(l)>0$ for $0<l \leq 2 \pi$ and $\lim _{l \rightarrow 0^{+}} w(l)=w(0)=0$." Now we define

$$
H_{z}^{(w)}=\left\{g \in L^{z}[0,2 \pi]: \sup _{l \neq 0} \frac{\|g(\cdot,+l)-g(\cdot)\|_{z}}{w(l)}<\infty, z \geq 1\right\}
$$

and

$$
\|\cdot\|_{z}^{(w)}=\|g\|_{z}^{(w)}=\|g\|_{z}+\sup _{l \neq 0} \frac{\|g(\cdot+l)-g(\cdot)\|_{z}}{w(l)} ; \quad z \geq 1 .
$$

Note $1 w(l)$ and $v(l)$ denote "Zygmund moduli of continuity [14]."

If we consider $\frac{w(l)}{v(l)}$ as positive and non-decreasing,

$$
\|g\|_{z}^{(v)} \leq \max \left(1, \frac{w(2 \pi)}{v(2 \pi)}\right)\|g\|_{z}^{(w)}<\infty
$$

Thus,

$$
H_{z}^{(w)} \subset H_{z}^{(v)} \subset L^{z} ; \quad z \geq 1
$$

\section{Remark 3}

(i) If $w(l)=l^{\alpha}$ in $H^{(w)}, H^{(w)}$ implies $H_{\alpha}$ class.

(ii) If $w(l)=l^{\alpha}$ in $H_{z}^{(w)}, H^{(w)}$ implies $H_{\alpha, z}$ class.

(iii) If $z \rightarrow \infty$ in $H_{z}^{(w)}, H_{z}^{(w)}$ implies $H^{(w)}$ class and $H_{\alpha, z}$ class implies $H_{\alpha}$ class.

Remark 4 We are not representing here the F. S. and C. F. S. as these trigonometric series are well known and the detailed work on these series can be found in [14].

We denote the $r$ th partial sum of the F. S. as

$$
s_{r}(g ; x)-g(x)=\frac{1}{2 \pi} \int_{0}^{\pi} \phi_{x}(l) \frac{\sin \left(r+\frac{1}{2}\right) l}{\sin \frac{l}{2}} d l .
$$

The $r$ th partial sum of C. F. S. is defined as

$$
s_{r}(\tilde{g} ; x)-\tilde{g}(x)=\frac{1}{2 \pi} \int_{0}^{\pi} \psi_{x}(l) \frac{\cos \left(r+\frac{1}{2}\right) l}{\sin \left(\frac{l}{2}\right)} d l,
$$


where

$$
\tilde{g}=-\frac{1}{2 \pi} \int_{0}^{\pi} \psi_{x}(l) \cot \left(\frac{l}{2}\right) d l
$$

"The error estimation of function $g$ is given by

$$
E_{r}(g)=\min \left\|g-t_{r}\right\|_{z}
$$

where $t_{r}$ is a trigonometric polynomial of degree $r$ [14]."

We write

$$
\begin{aligned}
& \phi_{x}(l)=\phi(x, l)=g(x+l)+g(x-l)-2 g(x), \\
& \psi_{x}(l)=\psi(x, l)=g(x+l)-g(x-l), \\
& \Delta p_{m}=p_{m}-p_{m+1}, \quad m \geq 0, \\
& H_{r}(l)=\frac{1}{2 \pi} \sum_{m=0}^{r} a_{r, m} \frac{1}{m+1} \sum_{v=0}^{m} \frac{\sin \left(v+\frac{1}{2}\right) l}{\sin \left(\frac{l}{2}\right)}, \\
& \tilde{H}_{r}(l)=\frac{1}{2 \pi} \sum_{m=0}^{r} a_{r, m} \frac{1}{m+1} \sum_{v=0}^{m} \frac{\cos \left(v+\frac{1}{2}\right) l}{\sin \left(\frac{l}{2}\right)} .
\end{aligned}
$$

\section{Main theorems}

Theorem 2.1 If $g \in H_{z}^{(w)}$ class; $z \geq 1$ and $\frac{w(l)}{v(l)}$ are positive and non-decreasing, then the error estimation of $g$ by $T C^{1}$ means of $F$. $S$. is

$$
\left\|t_{r}^{T . C^{1}}-g\right\|_{z}^{(v)}=O\left(\frac{1}{r+1} \int_{\frac{1}{r+1}}^{\pi} \frac{w(l)}{l^{2} v(l)} d l\right)
$$

where $T=\left(a_{r, m}\right)$ is an infinite triangular matrix satisfying (1) and $w, v$ are defined as in Note 1 provided

$$
\sum_{m=0}^{r-1}\left|\Delta a_{r, m}\right|=O\left(\frac{1}{r+1}\right) \quad \text { and } \quad(r+1) a_{r, r}=O(1) .
$$

Theorem 2.2 If $\tilde{g} \in H_{z}^{(w)}$ class; $z \geq 1$ and $\frac{w(l)}{v(l)}$ are positive and non-decreasing, then the error estimation of $\tilde{g}$ by $T C^{1}$ means of C. F. S. is

$$
\left\|\tilde{t}_{r}^{T \cdot C^{1}}-\tilde{g}\right\|_{z}^{(v)}=O\left(\frac{(\log (r+1)+1)}{r+1} \int_{\frac{1}{r+1}}^{\pi} \frac{w(l)}{l^{2} v(l)} d l\right)
$$

where $T=\left(a_{r, m}\right)$ is an infinite triangular matrix satisfying (1), (6) and $w$, $v$ are defined as in Note 1.

\section{Lemmas}

Lemma 3.1 Under condition (1), $H_{r}(l)=O(r+1)$ for $0<l<\frac{1}{r+1}$. 
Proof For $0<l<\frac{1}{r+1}, \sin \left(\frac{l}{2}\right) \geq \frac{l}{\pi}, \sin (r l) \leq r l$.

$$
\begin{aligned}
H_{r}(l)= & \frac{1}{2 \pi} \sum_{m=0}^{r} a_{r, m} \frac{1}{m+1} \sum_{v=0}^{m} \frac{\sin \left(v+\frac{1}{2}\right) l}{\sin \left(\frac{l}{2}\right)} \\
\left|H_{r}(l)\right| & \leq \frac{1}{2 \pi} \times \frac{\pi}{l}\left|\sum_{m=0}^{r} a_{r, m} \frac{1}{m+1} \sum_{v=0}^{m} \sin \left(v+\frac{1}{2}\right) l\right| \\
& =\frac{1}{2 l}\left|\sum_{m=0}^{r} a_{r, m} \frac{1}{m+1} \sum_{v=0}^{m} \sin (2 v+1) \frac{l}{2}\right| \\
& \leq \frac{1}{2 l}\left|\sum_{m=0}^{r} a_{r, m} \frac{1}{m+1} \sum_{v=0}^{m}(2 v+1) \frac{l}{2}\right| \\
& =\frac{1}{4}\left|\sum_{m=0}^{r} a_{r, m} \frac{1}{m+1} \sum_{v=0}^{m}(2 v+1)\right| \\
& =\frac{1}{4}\left|\sum_{m=0}^{r} a_{r, m} \frac{1}{m+1} \times(m+1)^{2}\right| \\
& =\frac{1}{4}\left|\sum_{m=0}^{r} a_{r, m}(m+1)\right| \\
& =\frac{1}{4}(m+1) \sum_{m=0}^{r}\left|a_{r, m}\right| \\
& =O(r+1) .
\end{aligned}
$$

Lemma 3.2 Under conditions (1) and (6), $H_{r}(l)=O\left(\frac{1}{(r+1) l^{2}}\right)$ for $\frac{1}{r+1} \leq l \leq \pi$.

Proof For $\frac{1}{r+1} \leq l \leq \pi, \sin \left(\frac{l}{2}\right) \geq \frac{l}{\pi}, \sin ^{2} r l \leq 1$ and using Abel's lemma, we have

$$
\begin{aligned}
H_{r}(l)= & \frac{1}{2 \pi} \sum_{m=0}^{r} a_{r, m} \frac{1}{r+1} \sum_{v=0}^{r} \frac{\sin \left(v+\frac{1}{2}\right) l}{\sin \left(\frac{l}{2}\right)}, \\
\left|H_{r}(l)\right| & \leq \frac{1}{2 \pi} \times \frac{\pi}{l}\left|\sum_{m=0}^{r} a_{r, m} \frac{1}{m+1} \sum_{v=0}^{m} \sin \left(v+\frac{1}{2}\right) l\right| \\
& =\frac{1}{2 l}\left|\sum_{m=0}^{r} a_{r, m} \frac{1}{m+1} \operatorname{Im}\left\{\sum_{v=0}^{m} e^{i\left(v+\frac{1}{2}\right) l}\right\}\right| \\
& =\frac{1}{2 l}\left|\sum_{m=0}^{r} a_{r, m} \frac{1}{m+1} \operatorname{Im}\left\{e^{i \frac{l}{2}} \sum_{v=0}^{m} e^{i v l}\right\}\right| \\
& =\frac{1}{2 l}\left|\sum_{m=0}^{r} a_{r, m} \frac{1}{m+1} \operatorname{Im}\left\{e^{\frac{i l}{2}} \frac{1-e^{i(m+1) l}}{1-e^{i l}}\right\}\right| \\
& =\frac{1}{2 l}\left|\sum_{m=0}^{r} a_{r, m} \frac{1}{m+1} \operatorname{Im}\left\{\frac{e^{i(m+1) l}-1}{2 i \sin \left(\frac{l}{2}\right)}\right\}\right| \\
& \leq \frac{1}{2 l} \times \frac{\pi}{l}\left|\sum_{m=0}^{r} a_{r, m} \frac{1}{m+1} \sin ^{2}(m+1) \frac{l}{2}\right|
\end{aligned}
$$




$$
\begin{aligned}
& \leq \frac{\pi}{2 l^{2}}\left|\sum_{m=0}^{r} a_{r, m} \frac{1}{m+1}\right| \\
& =\frac{\pi}{2 l^{2}}\left|\sum_{m=0}^{r-1}\left(a_{r, m}-a_{r, m+1}\right) \sum_{v=0}^{m} \frac{1}{v+1}+a_{r, r} \sum_{m=0}^{r} \frac{1}{m+1}\right| \\
& \leq \frac{\pi}{2 l^{2}}\left|\sum_{m=0}^{r-1} \Delta a_{r, m} \sum_{v=0}^{m} \frac{1}{v+1}\right|+a_{r, r}\left|\sum_{m=0}^{r} \frac{1}{m+1}\right| \\
& \leq \frac{\pi}{2 l^{2}}\left[\sum_{m=0}^{r-1}\left|\Delta a_{r, m}\right|+a_{r, r}\right] \max _{0 \leq m \leq d}\left|\sum_{m=0}^{d} \frac{1}{m+1}\right| \\
& =O\left(\frac{1}{(r+1) l^{2}}\right) .
\end{aligned}
$$

Lemma 3.3 Under condition (1), $\tilde{H}_{r}(l)=O\left(\frac{1}{l}\right)$ for $0<l<\frac{1}{r+1}$.

Proof For $0<l \leq \frac{1}{r+1}$, using $\sin \left(\frac{l}{2}\right) \geq \frac{l}{\pi}$ and $|\cos r l| \leq 1$, we obtain

$$
\begin{aligned}
\tilde{H}_{r}(l)= & \frac{1}{2 \pi} \sum_{m=0}^{r} a_{r, m} \frac{1}{m+1} \sum_{v=0}^{m} \frac{\cos \left(v+\frac{1}{2}\right) l}{\sin \left(\frac{l}{2}\right)} \\
\left|\tilde{H}_{r}(l)\right| & \leq \frac{1}{2 \pi} \times \frac{\pi}{l} \sum_{m=0}^{r} a_{r, m} \frac{1}{m+1} \sum_{v=0}^{m}\left|\cos \left(v+\frac{1}{2}\right) l\right| \\
& \leq \frac{1}{2 l} \sum_{m=0}^{r} a_{r, m} \frac{1}{m+1} \sum_{v=0}^{m} 1 \\
& \leq \frac{1}{2 l} \sum_{m=0}^{r} a_{r, m}, \\
\therefore \tilde{H}_{r}(l) & =O\left(\frac{1}{l}\right) .
\end{aligned}
$$

Lemma 3.4 Under conditions (1) and (6), $\tilde{H}_{r}(l)=O\left(\frac{1}{(r+1) l^{2}}\right)$ for $\frac{1}{r+1} \leq l \leq \pi$.

Proof For $\frac{1}{r+1} \leq l \leq \pi$, using $\sin \left(\frac{l}{2}\right) \geq \frac{l}{\pi}$, Abel's lemma, and $\left|\sum_{m=0}^{r} \frac{\sin (m+1) l}{m+1}\right| \leq 1+\frac{\pi}{2} \forall r$ and $l$ [15], we get

$$
\begin{aligned}
& \left|\tilde{H}_{r}(l)\right| \\
& \quad \leq \frac{1}{2 \pi} \times \frac{\pi}{l}\left|\sum_{m=0}^{r} a_{r, m} \frac{1}{m+1} \sum_{v=0}^{m} \cos \left(v+\frac{1}{2}\right) l\right| \\
& \quad \leq \frac{1}{2 l}\left|\sum_{m=0}^{r} a_{r, m} \frac{1}{m+1}\left\{\frac{2 \sin \left(\frac{l}{2}\right) \cos \frac{l}{2}+2 \sin \left(\frac{l}{2}\right) \cos \frac{3 l}{2}+\cdots+2 \sin \left(\frac{l}{2}\right) \cos \left(\frac{(2 m+1) l}{2}\right)}{2 \sin \left(\frac{l}{2}\right)}\right\}\right| \\
& \leq \frac{1}{4 l} \times \frac{\pi}{l} \mid \sum_{m=0}^{r} a_{r, m} \frac{1}{m+1}\{\sin l+\sin 2 l-\sin l+\sin 3 l-\sin 2 l+\cdots \\
& \quad+\sin (m+1) l-\sin m l\} \mid
\end{aligned}
$$




$$
\begin{aligned}
& \leq \frac{\pi}{4 l^{2}}\left|\sum_{m=0}^{r} a_{r, m} \frac{\sin (m+1) l}{m+1}\right| \\
& \leq \frac{\pi}{4 l^{2}}\left|\sum_{m=0}^{r-1}\left(a_{r, m}-a_{r, m+1}\right) \sum_{v=0}^{m} \frac{\sin (v+1) l}{v+1}+a_{r, r} \sum_{m=0}^{r} \frac{\sin (m+1) l}{m+1}\right| \\
& \leq \frac{\pi}{4 l^{2}}\left[\sum_{m=0}^{r-1}\left|\Delta a_{r, m}\right|\left|\sum_{v=0}^{m} \frac{\sin (v+1) l}{v+1}\right|+a_{r, r}\left|\sum_{m=0}^{r} \frac{\sin (m+1) l}{m+1}\right|\right] \\
& \leq\left[\frac{1}{l^{2}}\left(\sum_{m=0}^{r-1}\left|\Delta a_{r, m}\right|+a_{r, r}\right)\right] . \\
& =\left[\frac{1}{l^{2}}\left\{O\left(\frac{1}{r+1}\right)+O\left(\frac{1}{r+1}\right)\right\}\right] \\
& =O\left(\frac{1}{(r+1) l^{2}}\right) .
\end{aligned}
$$

Lemma 3.5 “([16], p. 93)” Let $g \in H_{z}{ }^{(w)}$, then for $0<l \leq \pi$ :

(i) $\|\phi(\cdot, l)\|_{z}=O(w(l))$;

(ii) $\|\phi(\cdot+y, l)-\phi(\cdot, l)\|_{z}=\left\{\begin{array}{l}O(w(l)) \\ O(w(|y|))\end{array} ;\right.$

(iii) If $w(l)$ and $v(l)$ are defined as in Note 1 , then $\|\phi(\cdot+y, l)-\phi(\cdot, l)\|_{z}=O\left(v(|y|)\left(\frac{w(l)}{v(l)}\right)\right)$.

Lemma 3.6 Let $\tilde{g} \in H_{z}{ }^{(w)}$, then for $0<l \leq \pi$ :

(i) $\|\psi(\cdot, l)\|_{z}=O(w(l))$;

(ii) $\|\psi(\cdot+y, l)-\psi(\cdot, l)\|_{z}=\left\{\begin{array}{l}O(w(l)) \\ O(w(|y|))\end{array}\right.$

(iii) If $w(l)$ and $v(l)$ are defined as in Note 1 , then $\|\psi(\cdot+y, l)-\psi(\cdot, l)\|_{z}=O\left(v(|y|)\left(\frac{w(l)}{v(l)}\right)\right)$.

Proof This lemma can be proved along the same lines as the proof of Lemma 3.5(iii).

\section{Proof of the main theorems}

\subsection{Proof of Theorem 2.1}

Proof Following Titchmarsh [17], $s_{r}(g ; x)$ of F. S. is given by

$$
s_{r}(g ; x)-g(x)=\frac{1}{2 \pi} \int_{0}^{\pi} \phi_{x}(l) \frac{\sin \left(m+\frac{1}{2}\right) l}{\sin \left(\frac{l}{2}\right)} d l .
$$

Now, denoting T.C $C^{1}$ transform of $s_{r}(g ; x)$ by $t_{r}^{T . C^{1}}$,

$$
\begin{aligned}
t_{r}^{T . C^{1}}(x)-g(x) & =\sum_{m=0}^{r} a_{r, m}\left(C_{m}^{1}(x)-g(x)\right) \\
& =\sum_{m=0}^{r} a_{r, m}\left(\frac{1}{m+1} \sum_{v=0}^{m} s_{v}(g ; x)-g(x)\right) \\
& =\int_{0}^{\pi} \phi_{x}(l)\left(\frac{1}{2 \pi} \sum_{m=0}^{r} a_{r, m} \frac{1}{m+1} \sum_{v=0}^{m} \frac{\sin \left(v+\frac{1}{2}\right) l}{\sin \left(\frac{l}{2}\right)}\right) d l, \\
t_{r}{ }^{T . C^{1}}(x)-g(x) & =\int_{0}^{\pi} \phi_{x}(l) H_{r}(l) d l .
\end{aligned}
$$


Let

$$
R_{r}(x)=t_{r}^{T \cdot C^{1}}(x)-g(x)=\int_{0}^{\pi} \phi_{x}(l) H_{r}(l) d l .
$$

Then

$$
R_{r}(x+y)-R_{r}(x)=\int_{0}^{\pi}(\phi(x+y, l)-\phi(x, l)) H_{r}(l) d l .
$$

“Using generalized Minkowski’s inequality Chui [18]," we get

$$
\begin{aligned}
\left\|R_{r}(\cdot,+y)-R_{r}(\cdot)\right\|_{z} & \leq \int_{0}^{\pi}\|\phi(\cdot+y, l)-\phi(\cdot, l)\|_{z} H_{r}(l) d t \\
& =\left(\int_{0}^{\frac{1}{r+1}}+\int_{\frac{1}{r+1}}^{\pi}\right)\|\phi(\cdot+y, l)-\phi(\cdot, l)\|_{z} H_{r}(l) d l \\
& =I_{1}+I_{2} .
\end{aligned}
$$

Using Lemmas 3.1 and 3.5(iii), we have

$$
\begin{aligned}
I_{1} & =\int_{0}^{\frac{1}{r+1}}\|\phi(\cdot+y, l)-\phi(\cdot, l)\|_{z} H_{r}(l) d l \\
& =O(r+1)\left(v(|y|) \int_{0}^{\frac{1}{r+1}} \frac{w(l)}{v(l)} d l\right) \\
& =O\left(v(|y|) \frac{w\left(\frac{1}{r+1}\right)}{v\left(\frac{1}{r+1}\right)}\right) .
\end{aligned}
$$

Also, using Lemmas 3.2 and 3.5(iii), we get

$$
\begin{aligned}
I_{2} & =\int_{\frac{1}{r+1}}^{\pi}\|\phi(\cdot+y, l)-\phi(\cdot, l)\|_{z} H_{r}(l) d l \\
& =O\left(\frac{1}{r+1} \int_{\frac{1}{r+1}}^{\pi} v(|y|) \frac{w(l)}{l^{2} v(l)} d l\right) .
\end{aligned}
$$

By (9), (10), and (11), we have

$$
\sup _{y \neq 0} \frac{\left\|R_{r}(\cdot,+y)-R_{r}(\cdot)\right\|_{z}}{v(|y|)}=O\left(\frac{w\left(\frac{1}{r+1}\right)}{v\left(\frac{1}{r+1}\right)}\right)+O\left(\frac{1}{r+1} \int_{\frac{1}{r+1}}^{\pi} \frac{w(l)}{l^{2} v(l)} d l\right) .
$$

Again applying Minkowski's inequality, Lemma 3.1, Lemma 3.2, and $\|\phi(\cdot, l)\|_{z}=O(w(l))$, we obtain

$$
\begin{aligned}
\left\|R_{r}(\cdot)\right\|_{z} & =\left\|t_{r}^{T \cdot C^{1}}-g\right\|_{z} \\
& \leq\left(\int_{0}^{\frac{1}{r+1}}+\int_{\frac{1}{r+1}}^{\pi}\right)\|\phi(\cdot, l)\|_{z} H_{r}(l) d l
\end{aligned}
$$




$$
\begin{aligned}
& =O\left((r+1) \int_{0}^{\frac{1}{r+1}} w(l) d l\right)+O\left(\frac{1}{r+1} \int_{\frac{1}{r+1}}^{\pi} \frac{w(l)}{l^{2}} d l\right) \\
& =O\left(w\left(\frac{1}{r+1}\right)\right)+O\left(\frac{1}{r+1} \int_{\frac{1}{r+1}}^{\pi} \frac{w(l)}{l^{2}} d l\right) .
\end{aligned}
$$

Now, we have

$$
\left\|R_{r}(\cdot)\right\|_{z}^{v}=\left\|R_{r}(\cdot)\right\|_{z}+\sup _{y \neq 0} \frac{\left\|R_{r}(\cdot,+y)-R_{r}(\cdot)\right\|_{z}}{v(|y|)} .
$$

Using (12) and (13), we get

$$
\begin{aligned}
\left\|R_{r}(\cdot)\right\|_{z}^{v}= & O\left(w\left(\frac{1}{r+1}\right)\right)+O\left(\frac{1}{r+1} \int_{\frac{1}{r+1}}^{\pi} \frac{w(l)}{l^{2}} d l\right) \\
& +O\left(\frac{w\left(\frac{1}{r+1}\right)}{v\left(\frac{1}{r+1}\right)}\right)+O\left(\frac{1}{r+1} \int_{\frac{1}{r+1}}^{\pi} \frac{w(l)}{l^{2} v(l)} d l\right) .
\end{aligned}
$$

By the monotonicity of $v(l), w(l)=\frac{w(l)}{v(l)} v(l) \leq v(\pi) \frac{w(l)}{v(l)}$ for $0<l \leq \pi$, we get

$$
\left\|R_{r}(\cdot)\right\|_{z}^{v}=O\left(\frac{w\left(\frac{1}{r+1}\right)}{v\left(\frac{1}{r+1}\right)}\right)+O\left(\frac{1}{r+1} \int_{\frac{1}{r+1}}^{\pi} \frac{w(l)}{l^{2} v(l)} d l\right)
$$

Since $w$ and $v$ are moduli of continuity such that $\frac{w(l)}{v(l)}$ is positive and non-decreasing, therefore

$$
\frac{1}{r+1} \int_{\frac{1}{r+1}}^{\pi} \frac{w(l)}{l^{2} v(l)} d l \geq \frac{w\left(\frac{1}{r+1}\right)}{v\left(\frac{1}{r+1}\right)}\left(\frac{1}{r+1}\right) \int_{\frac{1}{r+1}}^{\pi} \frac{1}{l^{2}} d l \geq \frac{w\left(\frac{1}{r+1}\right)}{2 v\left(\frac{1}{r+1}\right)}
$$

Then

$$
\frac{w\left(\frac{1}{r+1}\right)}{v\left(\frac{1}{r+1}\right)}=O\left(\frac{1}{r+1} \int_{\frac{1}{r+1}}^{\pi} \frac{w(l)}{l^{2} v(l)} d l\right)
$$

From (16) and (17), we get

$$
\begin{aligned}
& \left\|R_{r}(\cdot)\right\|_{z}^{(v)}=O\left(\frac{1}{r+1} \int_{\frac{1}{r+1}}^{\pi} \frac{w(l)}{l^{2} v(l)} d l\right), \\
& \left\|t_{r}^{T \cdot C^{1}}-g\right\|_{z}^{(v)}=O\left(\frac{1}{r+1} \int_{\frac{1}{r+1}}^{\pi} \frac{w(l)}{l^{2} v(l)} d l\right) .
\end{aligned}
$$

\subsection{Proof of Theorem 2.2}

Proof The integral representation of $s_{r}(\tilde{g} ; x)$ is given by

$$
s_{r}(\tilde{g} ; x)-\tilde{g}(x)=\frac{1}{2 \pi} \int_{0}^{\pi} \psi_{x}(l) \frac{\cos \left(r+\frac{1}{2}\right) l}{\sin \left(\frac{l}{2}\right)} d l
$$


Now, denoting T.C. $C^{1}$ transform of $s_{r}(\tilde{g} ; x)$ by $\tilde{t}_{r}^{T . C^{1}}$, we get

$$
\begin{aligned}
\tilde{t}_{r}^{T . C^{1}}(x)-\tilde{g}(x) & =\sum_{m=0}^{r} a_{r, m}\left(C_{m}^{1}(x)-\tilde{g}(x)\right) \\
& =\sum_{m=0}^{r} a_{r, m}\left(\frac{1}{m+1} \sum_{v=0}^{m} s_{\nu}(\tilde{g} ; x)-\tilde{g}(x)\right) \\
& =\int_{0}^{\pi} \psi_{x}(l)\left(\frac{1}{2 \pi} \sum_{m=0}^{r} a_{r, m} \frac{1}{m+1} \sum_{v=0}^{m} \frac{\cos \left(v+\frac{1}{2}\right)}{\sin \left(\frac{l}{2}\right)}\right) d l, \\
\tilde{t}_{r}^{T . C^{1}}(x)-\tilde{g}(x) & =\int_{0}^{\pi} \psi_{x}(l) \tilde{H}_{r}(l) d l .
\end{aligned}
$$

Let

$$
\tilde{R}_{r}(x)=\tilde{t}_{r}^{T \cdot C^{1}}(x)-\tilde{g}(x)=\int_{0}^{\pi} \psi_{x}(l) \tilde{H}_{r} d l
$$

Then

$$
\tilde{R}_{r}(x+y)-\tilde{R}_{r}(x)=\int_{0}^{\pi}\left\{\psi_{x}(x+y, l)-\psi_{x}(x, l)\right\} \tilde{H}_{r}(l) d l .
$$

Using "generalized Minkowski’s inequality Chui [18]," we get

$$
\begin{aligned}
\left\|\tilde{R}_{r}(\cdot+y)-\tilde{R}_{r}(\cdot)\right\|_{z} & \leq \int_{0}^{\pi}\left\|\psi_{x}(\cdot+y, l)\right\|_{z} \tilde{H}_{r}(l) d l \\
& =\left(\int_{0}^{\frac{1}{r+1}}+\int_{\frac{1}{r+1}}^{\pi}\right)\|\psi(\cdot+y, l)-\psi(\cdot, l)\|_{z} \tilde{R}_{r}(l) d l \\
& =I_{1}+I_{2} .
\end{aligned}
$$

Using Lemmas 3.3 and 3.6(iii), we have

$$
\begin{aligned}
I_{1} & =\int_{0}^{\frac{1}{r+1}}\|\psi(\cdot+y, l)-\psi(\cdot, l)\|_{z} \tilde{H}_{r}(l) d l \\
& =O\left(v(|y|) \frac{w\left(\frac{1}{r+1}\right)}{v\left(\frac{1}{r+1}\right)} \int_{0}^{\frac{1}{r+1}} \frac{1}{l} d l\right) \\
& =O\left(v(|y|) \frac{w\left(\frac{1}{r+1}\right)}{v\left(\frac{1}{r+1}\right)} \log (r+1)\right) .
\end{aligned}
$$

Again using Lemmas 3.4 and 3.6(iii), we have

$$
\begin{aligned}
I_{2} & =\int_{\frac{1}{r+1}}^{\pi}\|\psi(\cdot+y, l)-\psi(\cdot, l)\|_{z} \tilde{H}_{r}(l) d l \\
& =O\left(\frac{1}{r+1} \int_{\frac{1}{r+1}}^{\pi} v(|y|) \frac{w(l)}{l^{2} v(l)} d l\right) .
\end{aligned}
$$


Using (19), (20), and (21), we have

$$
\sup _{y \neq 0} \frac{\left\|\tilde{R}_{r}(\cdot+y)-\tilde{R}_{r}(\cdot)\right\|_{z}}{v(|y|)}=O\left(\frac{w\left(\frac{1}{r+1}\right)}{v\left(\frac{1}{r+1}\right)} \log (r+1)\right)+O\left(\frac{1}{r+1} \int_{\frac{1}{r+1}}^{\pi} \frac{w(l)}{l^{2} v(l)} d l\right) .
$$

Again applying Minkowski's inequality, Lemma 3.3, Lemma 3.4, and $\|\psi(\cdot, l)\|_{z}=O(w(l))$, we have

$$
\begin{aligned}
\left\|\tilde{R}_{r}(\cdot)\right\|_{z} & =\left\|\tilde{t}_{r}^{T \cdot C^{1}}-\tilde{g}\right\|_{z} \leq\left(\int_{0}^{\frac{1}{r+1}}+\int_{\frac{1}{r+1}}^{\pi}\right)\|\psi(\cdot, l)\|_{z} \tilde{H}_{r}(l) d l \\
& =O\left(\int_{0}^{\frac{1}{r+1}} \frac{w(l)}{l} d l\right)+O\left(\frac{1}{r+1} \int_{\frac{1}{r+1}}^{\pi} \frac{w(l)}{l^{2}} d l\right) \\
& =O\left(w\left(\frac{1}{r+1}\right) \log (r+1)\right)+O\left(\frac{1}{r+1} \int_{\frac{1}{r+1}}^{\pi} \frac{w(l)}{l^{2}} d l\right) .
\end{aligned}
$$

Now, we have

$$
\left\|\tilde{R}_{r}(\cdot)\right\|_{z}^{(v)}=\left\|\tilde{R}_{r}(\cdot)\right\|_{z}+\sup _{y \neq 0} \frac{\left\|\tilde{R}_{r}(\cdot+y)-\bar{R}_{r}(\cdot)\right\|_{z}}{v(|y|)}
$$

Using (22) and (23), we get

$$
\begin{aligned}
\left\|\tilde{R}_{r}(\cdot)\right\|_{z}^{(v)}= & O\left((\log (r+1)) w\left(\frac{1}{r+1}\right)\right)+O\left(\frac{1}{r+1} \int_{\frac{1}{r+1}}^{\pi} \frac{w(l)}{l^{2}} d l\right) \\
& +O\left(\frac{w\left(\frac{1}{r+1}\right)}{v\left(\frac{1}{r+1}\right)} \log (r+1)\right)+O\left(\frac{1}{r+1} \int_{\frac{1}{r+1}}^{\pi} \frac{w(l)}{l^{2} v(l)} d l\right) .
\end{aligned}
$$

By the monotonicity of $v(l)$, we have $w(l)=\frac{w(l)}{v(l)} v(l) \leq v(\pi) \frac{w(l)}{v(l)}, 0<l \leq \pi$, we get

$$
\left\|\tilde{R}_{r}(\cdot)\right\|_{z}^{(v)}=O\left(\frac{w\left(\frac{1}{r+1}\right)}{v\left(\frac{1}{r+1}\right)} \log (r+1)\right)+O\left(\frac{1}{r+1} \int_{\frac{1}{r+1}}^{\pi} \frac{w(l)}{l^{2} v(l)} d l\right) .
$$

Using the fact that $\frac{w(l)}{v(l)}$ is positive and non-decreasing, we have

$$
\begin{aligned}
\frac{1}{r+1} \int_{\frac{1}{r+1}}^{\pi} \frac{w(l)}{l^{2} v(l)} d l & \geq \frac{w\left(\frac{1}{r+1}\right)}{v\left(\frac{1}{r+1}\right)} \frac{1}{r+1} \int_{\frac{1}{r+1}}^{\pi} \frac{1}{l^{2}} d l \\
& \geq \frac{w\left(\frac{1}{r+1}\right)}{2 v\left(\frac{1}{r+1}\right)} .
\end{aligned}
$$

Then

$$
\frac{w\left(\frac{1}{r+1}\right)}{v\left(\frac{1}{r+1}\right)}=O\left(\frac{1}{r+1} \int_{\frac{1}{r+1}}^{\pi} \frac{w(l)}{l^{2} v(l)} d l\right)
$$


From (24) and (25), we get

$$
\begin{aligned}
& \left\|\tilde{R}_{r}(\cdot)\right\|_{z}^{(v)}=O\left(\frac{\log (r+1)}{r+1} \int_{\frac{1}{r+1}}^{\pi} \frac{w(l)}{l^{2} v(l)} d l\right)+O\left(\frac{1}{r+1} \int_{\frac{1}{r+1}}^{\pi} \frac{w(l)}{l^{2} v(l)} d l\right), \\
& \therefore\left\|\tilde{t}_{r}^{T \cdot C^{1}}-\tilde{g}\right\|_{z}^{(v)}=O\left(\frac{\log (r+1)+1}{r+1} \int_{\frac{1}{r+1}}^{\pi} \frac{w(l)}{l^{2} v(l)} d l\right) .
\end{aligned}
$$

\section{Corollary}

Corollary 5.1 Let $0 \leq \beta<\alpha \leq 1$ and $\tilde{g} \in H_{(\alpha), z} ; z \geq 1$. Then

$$
\left\|\tilde{t}_{r}^{T . C^{1}}-\tilde{g}\right\|_{(\beta), z}= \begin{cases}O\left[(\log (r+1) e)(r+1)^{\beta-\alpha}\right] & \text { if } 0 \leq \beta<\alpha<1 \\ O\left[\frac{(\log (r+1) e)(\log (r+1) \pi)}{r+1}\right] & \text { if } \beta=0, \alpha=1\end{cases}
$$

Proof Putting $w(l)=l^{\alpha}, v(l)=l^{\beta}, 0 \leq \beta<\alpha \leq 1$ in (26)

$$
\begin{aligned}
& \left\|\tilde{t}_{r}^{T . C^{1}}-\tilde{g}\right\|_{(\beta), z}=O\left[\frac{\log (r+1) e}{r+1} \int_{\frac{1}{r+1}}^{\pi} t^{\alpha-\beta-2} d l\right] \\
& \Rightarrow \quad\left\|\tilde{t}_{r}^{T . C^{1}}-\tilde{g}\right\|_{(\beta), z}= \begin{cases}O\left(\frac{(\log (r+1) e)}{(r+1)} \int_{\frac{1}{r+1}}^{\pi} l^{\alpha-\beta-2} d l\right) & \text { if } 0 \leq \beta<\alpha<1, \\
O\left(\frac{\log (r+1) e}{r+1} \int_{\frac{1}{r+1}}^{\pi} l^{-1} d l\right) & \text { if } \beta=0, \alpha=1,\end{cases} \\
& \therefore\left\|\tilde{t}_{r}^{T . C^{1}}-\tilde{g}\right\|_{(\beta), z}= \begin{cases}O\left[(\log (r+1) e)(r+1)^{\beta-\alpha}\right] & \text { if } 0 \leq \beta<\alpha<1, \\
O\left[\frac{(\log (r+1) e)}{r+1} \times \log (r+1) \pi\right] & \text { if } \beta=0, \alpha=1 .\end{cases}
\end{aligned}
$$

Corollary 5.2 Let $0 \leq \beta<\alpha \leq 1, a, b \in \mathbb{R}$ and suppose $w(l)=\frac{l^{\alpha}}{\left(\log \frac{1}{l}\right)^{a}}, w(l)=\frac{l^{\beta}}{\left(\log \frac{1}{l}\right)^{b}}, 0<l \leq$ $\pi, \tilde{g} \in H_{z}^{(w)}, z \geq 1$. Then

$$
\left\|\tilde{t}_{r}^{T . C^{1}}-\tilde{g}\right\|_{z}^{(v)}= \begin{cases}O\left[\frac{\log (r+1) e}{\{\log (r+1)\}^{b-a}}\right] & \text { if } \alpha=\beta \text { and } a-b \geq-1, \\ O\left[\frac{(\log (r+1) e)}{\log (r+1)}\right] & \text { if } \alpha=\beta \text { and } a-b=-1 .\end{cases}
$$

Proof We have

$$
\begin{aligned}
& \left\|\tilde{t}_{r}^{T . C^{1}}-\tilde{f}\right\|_{z}^{(v)}=O\left(\frac{\log (r+1) e}{r+1} \int_{\frac{1}{r+1}}^{\pi} \frac{l^{\alpha}}{l^{2}\left(\log \frac{1}{l}\right)^{a} \times \frac{l^{\beta}}{\left(\log \frac{1}{l}\right)^{b}}} d l\right) \\
& =O\left(\frac{\log (r+1) e}{r+1} \int_{\frac{1}{r+1}}^{\pi} l^{\alpha-\beta-2}\left(\log \frac{1}{l}\right)^{b-a} d l\right) \\
& \therefore\left\|\tilde{t}_{\eta}^{T . C^{1}}-\tilde{g}\right\|_{z}^{(v)}= \begin{cases}O\left[\frac{\log (r+1) e}{\{\log (r+1)\}^{b-a}}\right] & \text { if } \alpha=\beta \text { and } a-b \geq-1 . \\
O\left[\frac{(\log (r+1) e)}{\log (r+1)}\right] & \text { if } \alpha=\beta \text { and } a-b=-1 .\end{cases}
\end{aligned}
$$

Corollary 5.3 If $a_{r, m}=\frac{1}{(r-m+1) \log (r+1)}$, then T. $C^{1}$ means reduces to $\left(H, \frac{1}{r+1}\right)(C, 1)$ means and error estimation of a function $g \in H_{z}^{(w)}$ by $\left(H, \frac{1}{r+1}\right)(C, 1)$ means of $F$. S. is

$$
\left\|t_{r}^{H \cdot C^{1}}-g\right\|_{z}^{(v)}=O\left(\frac{1}{r+1} \int_{\frac{1}{r+1}}^{\pi} \frac{w(l)}{l^{2} v(l)} d l\right) .
$$


Corollary 5.4 If $a_{r, m}=\frac{p_{r-m}}{P_{r}}$, then T.C. $C^{1}$ means reduces to $N_{p} . C^{1}$ and the error estimation of $g \in H_{v}^{(w)}$ by $N_{p}$. $C^{1}$ means of $F$. S. is

$$
\left\|t_{r}^{N_{p} \cdot C^{1}}-g\right\|_{z}^{(v)}=O\left(\frac{1}{r+1} \int_{\frac{1}{r+1}}^{\pi} \frac{w(l)}{l^{2} v(l)} d l\right) .
$$

Corollary 5.5 If $a_{r, m}=\frac{p_{r-m} q_{m}}{R_{r}}$, then $T . C^{1}$ means reduces to $N_{p, q} . C^{1}$ and the error estimation of $g \in H_{v}^{(w)}$ by $N_{p, q} . C^{1}$ means of F. S. is

$$
\left\|t_{r}^{N_{p, q} \cdot C^{1}}-g\right\|_{z}^{(v)}=O\left(\frac{1}{r+1} \int_{\frac{1}{r+1}}^{\pi} \frac{w(l)}{l^{2} v(l)} d l\right) .
$$

Corollary 5.6 If $a_{r, m}=\frac{1}{(r-m+1) \log (r+1)}$, then T. $C^{1}$ means reduces to $\left(H, \frac{1}{r+1}\right)(C, 1)$ means and the error estimation of a function $\tilde{g} \in H_{z}^{(w)}$ by $\left(H, \frac{1}{r+1}\right)(C, 1)$ means of C. F. S. is

$$
\left\|\tilde{t}_{r}^{H \cdot C^{1}}-\tilde{g}\right\|_{z}^{(v)}=O\left(\frac{(\log (r+1)+1)}{r+1} \int_{\frac{1}{r+1}}^{\pi} \frac{w(l)}{l^{2} v(l)} d l\right) .
$$

Corollary 5.7 If $a_{r, m}=\frac{p_{r-m}}{P_{r}}$, then T.C. $C^{1}$ means reduces to $N_{p} \cdot C^{1}$ and the error estimation of $\tilde{g} \in H_{v}^{(w)}$ by $N_{p}$. $C^{1}$ means of C. F. S. is

$$
\left\|\tilde{t}_{r}^{N_{p} \cdot C^{1}}-\tilde{f}\right\|_{z}^{(v)}=O\left(\frac{(\log (r+1)+1)}{r+1} \int_{\frac{1}{r+1}}^{\pi} \frac{w(l)}{l^{2} v(l)} d l\right) .
$$

Corollary 5.8 If $a_{r, m}=\frac{p_{r-m} q_{m}}{R_{r}}$, then T.C. $C^{1}$ means reduces to $N_{p, q} \cdot C^{1}$ and the error estimation of $\tilde{f} \in H_{v}^{(w)}$ by $N_{p, q} . C^{1}$ means of C. F. S. is

$$
\left\|\tilde{t}_{r}^{N_{p, q} \cdot C^{1}}-\tilde{g}\right\|_{z}^{(v)}=O\left(\frac{(\log (r+1)+1)}{r+1} \int_{\frac{1}{r+1}}^{\pi} \frac{w(l)}{l^{2} v(l)} d l\right) .
$$

\section{Remark 5}

(i) If $z \rightarrow \infty$ in $H_{z}^{(w)}$ class, then $H_{z}^{(w)}$ class reduces to $H^{(w)}$ class. Also putting $w(l)=l^{\alpha}$ and $v(l)=l^{\beta}$ in our Theorem 2.1, $H^{(w)}$ class reduces to $H_{\alpha}$ class; then, by putting $\beta=0$ in $H_{\alpha}$ class, $H_{\alpha}$ class reduces to $\operatorname{Lip} \alpha$ class.

(ii) In our Theorem 2.1, by putting $w(l)=l^{\alpha}, v(l)=l^{\beta}$ in $H_{z}^{(w)}$ class, $H_{z}^{(w)}$ class reduces to $H_{\alpha, z}$; then, by putting $\beta=0$ in $H_{\alpha, z}$ class, $H_{\alpha, z}$ class reduces to $\operatorname{Lip}(\alpha, z)$ class.

\section{Particular cases}

6.1. Using Remark 4(i), our Theorem 2.1 becomes a particular case of Dhakal [1].

6.2. Using Remark 4(ii) and putting $a_{r, m}=\frac{p_{r-m} q_{m}}{R_{r}}$, where $R_{r}=\sum_{m=0}^{r} p_{\mu} q_{r-m}$ in our of Theorem 2.1, our result of Theorem 2.1 becomes a particular case of the main theorem of Kushwaha and Dhakal [3].

6.3. Using Remark 4(i) and putting $a_{r, m}=\frac{p_{r-m} q_{m}}{R_{r}}$, where $R_{r}=\sum_{m=0}^{r} p_{m} q_{r-m}$ in our Theorem 2.1, our Theorem 2.1 becomes a particular case of the main theorem of Dhakal [2]. 


\title{
7 Conclusion
}

Approximation by trigonometric polynomials is at the heart of approximation theory. Much of the advances in the theory of trigonometric approximation are due to the periodicity of the functions. The study of error approximation of periodic functions in Lipschitz and Hölder classes has been of great interest among the researchers [1-11], and [12] in recent past. The trigonometric Fourier approximation (TFA) is of great importance due to its wide applications in different branches of engineering such as electronics and communication engineering, electrical and electronics engineering, computer science engineering, etc. Several elegant results on TFA can be found in a monograph [14].

In this paper, we, for the first time, obtain the best approximation of the functions $g$ and $\tilde{g}$ in a generalized Hölder class $H_{r}^{(w)}(r \geq 1)$ using Matrix- $C^{1}\left(T . C^{1}\right)$ method of F. S. and C. F. S. respectively. Since, in view of Remark 2 , the product summability means $H . C^{1}$, $N_{p} C^{1}, N_{p, q} C^{1}$, and $\bar{N}_{p} C^{1}$ are the particular cases of Matrix- $C^{1}$ method, so our results also hold for these methods, which are represented in a form of corollaries. In view of Remark 1 , it has been shown that $\left(T C^{1}\right)$ method is more powerful than the individual $T$ method and $C^{1}$ method. Moreover, in view of Remark 5, some previous results (see Sect. 6) become the particular cases of our Theorem 2.1. We also deduce a corollary for the $H_{\alpha, r}$ class $(r \geq 1)$.

Some other studies regarding the modulus of continuity (smoothness) of functions using more generalized functional spaces may be addressed as a future work.

\begin{abstract}
Acknowledgements
The first author expresses his gratitude towards his mother for her blessings. The first author also expresses his gratitude towards his father in heaven, whose soul is always guiding and encouraging him. The second author is thankful to the University Grants Commission (India) for providing Junior Research Fellowship (JRF) to carry out the present work as a part of PhD degree. The second author also expresses his gratitude towards his parents for blessings and is very grateful to his guide Dr. H. K. Nigam without whose help he couldn't complete his work. Both the authors are also grateful to the Hon'ble vice-chancellor, Central University of South Bihar, for motivation to carry out this work.
\end{abstract}

Funding

Not applicable.

Competing interests

The authors declare that they have no competing interests.

Authors' contributions

HK framed the problems. HK and MH carried out the results and wrote the manuscript. All the authors read and approved the final manuscript.

\section{Publisher's Note}

Springer Nature remains neutral with regard to jurisdictional claims in published maps and institutional affiliations.

Received: 8 June 2018 Accepted: 27 September 2018 Published online: 11 October 2018

References

1. Dhakal, B.P.: Approximation of functions belonging to Lip $\alpha$ class by matrix-Cesàro summability method. Int. Math. Forum 5(35), 1729-1735 (2010)

2. Dhakal, B.P.: Approximation of a function $f$ belonging to Lip class by $(N, p, q) C_{1}$ means of its Fourier series. Int. J. Eng. Technol. 2(3), 1-15 (2013)

3. Kushwaha, J.K., Dhakal, B.P.: Approximation of a function belonging to $L i p(\alpha, r)$ class by $N_{p, q}$. $C_{1}$ summability method of its Fourier series. Nepal J. Sci. Technol. 14(2), 117-122 (2013)

4. Khatri, K.: Degree of approximation of functions $\tilde{f} \in H_{w}$ class by the $\left(N_{p} . E_{1}\right)$ means in the Hölder metric. Int. J. Math. Math. Sci. 2014, Article ID 837408 (2014)

5. Khatri, K., Mishra, V.N.: Degree of approximation by the T.E $E^{1}$ means of conjugate series of Fourier series in the Hölder metric. Iran. J. Sci. Technol., Trans. A Sci. (2017). https://doi.org/10.1007/s40995-017-0272-3

6. Mishra, L.N.: On existence and behaviour of solutions to some nonlinear integral equations with applications. Ph.D. thesis, National Institute of Technology, Silchar 788 010, Assam, India (2017)

7. Mishra, V.N., Khatri, K., Mishra, L.N., Deepmala: Trigonometric approximation of periodic signals belonging to generalized weighted Lipschitz $W\left(L_{r}, \xi(t)\right)(r \geq 1)$-class by Nörlund-Euler $\left(N, p_{n}\right)(E, q)$ operator of conjugate series of its Fourier series. J. Class. Anal. 5(2), 91-105 (2014). https://doi.org/10.7153/jca-05-08 
8. Mishra, L.N., Mishra, V.N., Khatri, K., Deepmala: On the trigonometric approximation of signals belonging to generalized weighted Lipschitz $W\left(L^{r}, \xi(t)\right)(r \geq 1)$-class by matrix $C^{1} . N_{p}$ operator of conjugate series of its Fourier series. Appl. Math. Comput. 237, 252-263 (2014)

9. Mishra, V.N., Khatri, K., Mishra, L.N.: Product $\left(N, p_{n}\right) C_{1}$ summability of a sequence of Fourier coefficients. Math. Sci. 6,38 (2012)

10. Mishra, V.N., Mishra, L.N.: Trigonometric approximation of signals (functions) in $L_{p}$ ( $p \geq 1$ )-norm. Int. J. Contemp. Math. Sci. 7(19), 909-918 (2012)

11. Mittal, M.L., Rhoades, B.E., Mishra, V.N., Singh, U.: Using infinite matrices to approximate functions of class Lip $(\alpha, p)$ using trigonometric polynomials. J. Math. Anal. Appl. 326, 667-676 (2007)

12. Tiwari, K.S., Bariwal, C.S.: The degree of approximation of functions in the Hölder metric by triangular matrix method of Fourier series. Int. J. Pure Appl. Math. 76(2), 227-232 (2012)

13. Töeplitz, O.: Uberallagemeine lineara Mittelbil. dunger. P.M.F. 22, 113-119 (1913)

14. Zygmund, A.: Trigonometric Series, 3rd edn. Cambridge University Press, Cambridge (2002)

15. Gronwall, T.H.: Über die Gibbssche Erscheinung und die trigonometrische summen $\sin x+(1 / 2) \sin 2 x+(1 / 3) \sin 3 x+\cdots+(1 / n) \sin n x$. Math. Ann. 72, 228-243 (1912)

16. Lal, S., Mishra, A.: The method of summation $(E, 1)\left(N, p_{n}\right)$ and trigonometric approximation of function in generalized Holder metric. J. Indian Math. Soc. 80(1-2), 87-98 (2013)

17. Titchmarsh, E.C.: The Theory of Functions. Oxford University Press, London (1939)

18. Chui, C.K.: An Introduction to Wavelets: Wavelet Analysis and Applications, vol. 1. Academic Press, San Diego (1992)

\section{Submit your manuscript to a SpringerOpen ${ }^{\circ}$ journal and benefit from:}

- Convenient online submission

Rigorous peer review

- Open access: articles freely available online

- High visibility within the field

- Retaining the copyright to your article

Submit your next manuscript at $\boldsymbol{~ s p r i n g e r o p e n . c o m ~}$ 